France; ${ }^{5}$ 1. Institut National de la Santé et de la Recherché Médicale, Villejuif, France; ${ }^{6}$ German Cancer Research Center, Heidelberg, Germany; ${ }^{7}$ Unit of Infections and Cancer, ICO and CIBERESP, Barcelona, Spain; ${ }^{8}$ Department of Cancer Epidemiology and Genetics, Brno, Czech Republic; ${ }^{9}$ School of Nursing and Human Sciences, Dublin City University, Dublin, Ireland; ${ }^{10}$ Dijon University Hospital, Dijon, France; ${ }^{11}$ Centre of Chronic Immunodeficiency, University of Freiburg, Freiburg, Germany; ${ }^{12}$ SPO Cancer Prevention and Research Institute, Florence, Italy; ${ }^{13}$ Centre for Public Health Research, Massey University, Wellington, New Zealand; ${ }^{14}$ International Agency for Research on Cancer, Lyon, France

\subsection{6/oemed-2013-101717.379}

Objectives To test the association between occupational exposure to trichloroethylene (TCE) and risk of non Hodgkin lymphoma (NHL), we conducted a pooled analysis of four international case-control studies.

Methods Studies were selected which included state-of-the art retrospective assessment of occupational exposure to TCE and histological information on lymphoma subtype. Overall, the pooled study population included 3788 NHL cases and 4279 controls. Summary indicators of exposure were harmonised across studies. We conducted unconditional logistic regression analysis to test the association between the harmonised TCE exposure estimates and NHL and its major subtypes, adjusting by age, gender, and study.

Results Among the total study population, risk of follicular lymphoma, but not NHL overall or other subtypes, increased by probability $(p=0.02)$ and intensity level $(p=0.04)$ of TCE exposure. When the analysis was restricted to subjects most likely exposed to TCE, risk of NHL overall $(p=0.009)$, follicular lymphoma $(p=0.04)$, and chronic lymphocytic leukaemia (CLL) $(p=0.01)$ showed a linear increase by duration of exposure. No heterogeneity in NHL risk associated with high probability of exposure to TCE (all intensity levels combined) was detected.

Conclusion With due caution because of several limitations, our pooled analysis supports the hypothesis of an increased risk of NHL, and particularly of the follicular lymphoma and CLL subtypes, associated with occupational exposure to TCE.

\section{MULTIPLE PESTICIDE EXPOSURES AND THE RISK OF MULTIPLE MYELOMA IN CANADIAN MEN}

${ }^{1} \mathrm{~L}$ K Kachuri, ${ }^{2}$ Demers, ${ }^{2}$ Blair, ${ }^{3}$ Spinelli, ${ }^{1}$ Pahwa, ${ }^{4}$ McLaughlin, ${ }^{5}$ Dosman, ${ }^{6}$ Pahwa, ${ }^{7}$ Harris. 'Occupational Cancer Research Centre, Cancer Care Ontario, Toronto, Canada; ${ }^{2}$ Occupational Cancer Research Centre, Toronto, ON, Canada; ${ }^{3} B C$ Cancer Agency, Vancouver BC, Canada; ${ }^{4}$ Samuel Lunenfeld Research Institute, Toronto, ON, Canada; ${ }^{5}$ Canadian Centre for Health and Safety in Agriculture, University of Saskatchewan, Saskatoon, SK, Canada; ${ }^{6}$ Department of Community Health and Epidemiology, University of Saskatchewan, Saskatoon, SK, Canada; ${ }^{7}$ Cancer Care Ontario, Toronto, ON, Canada

\subsection{6/oemed-2013-101717.380}

Objectives Multiple myeloma (MM) has been linked to certain agricultural exposures, including pesticides, however the effects of exposure to multiple pesticides have not been explored. This analysis investigated the association between self-reported use of multiple pesticides and MM risk. Commonly used pesticide combinations and interactive effects were also assessed.

Methods A frequency matched population-based case-control study was conducted among men in 6 Canadian provinces between 1991 and 1994. Data from 342 MM cases and 1506 controls were analysed using logistic regression to calculate odds ratios (OR) and 95\% confidence intervals (CI). Pesticides were grouped by type, chemical class and carcinogenicity. Carcinogenic probability values were created using evaluations from the
International Agency for Research on Cancer and U. S. Environmental Protection Agency. Regression models were adjusted for age, province of residence, use of proxy respondents, smoking, and selected medical history variables. Trends were examined using ordinal variables. Commonly used pesticide combinations were assessed for interaction on the additive scale using the interaction contrast ratio (ICR).

Results Multiple pesticide use was not associated with monotonically increasing odds of $\mathrm{MM}$, although positive trends were observed for "probably" carcinogenic pesticides $\left(p_{\text {trend }}=0.01\right)$, insecticides ( $\left.\mathrm{p}_{\text {trend }}=0.07\right)$, and fungicides $\left(\mathrm{p}_{\text {trend }}=0.05\right)$. Higher odds of MM were observed among men who reported using at least one carbamate pesticide (OR $=1.99$, 95\% CI: 1.19-3.33), one phenoxyherbicide $(\mathrm{OR}=1.60,95 \% \mathrm{CI}: 1.11-$ 2.30), 3 or more "probably" carcinogenic pesticides (OR = 2.14, 95\% CI: 1.01-4.52), and 3 or more organochlorines (OR $=2.26$, 95\% CI: 1.07-4.78). Investigating commonly used pesticide combinations, revealed increased odds among men who used both chlordane and mecoprop (OR $=2.18,95 \%$ CI: $1.12-$ 4.27; ICR $=0.63$ ).

Conclusions Focusing on multiple pesticides is important because this more accurately reflects how exposures occur in occupational settings. Although the overall pattern of results was complex, excess risks observed for certain pesticide types and chemical classes suggest these may be MM risk factors.

\section{ENDOCRINE DISRUPTORS AND THE RISK OF LYMPHOMA IN THE EPILYMPH STUDY}

${ }^{1}$ L C Costas, ${ }^{2}$ Infante-Rivard, ${ }^{3}$ Cocco, ${ }^{4}$ van Tongeren, ${ }^{5}$ Zock, ${ }^{1}$ de Sanjosé. ${ }^{1}$ Catalan Institute of Oncology, Barcelona, Spain; ${ }^{2}$ McGill University, Montreal, Canada; ${ }^{3}$ University of Cagliari, Cagliari, Italy; ${ }^{4}$ Institute of Occupational Medicine, Edinburgh, United Kingdom; ${ }^{5}$ Centre for Research in Environmental Epidemiology, Barcelona, Spain

\subsection{6/oemed-2013-101717.381}

Objectives Some industrial chemicals and pesticides might have endocrine disrupting effects. While some pesticides and solvents have been associated with an increased risk of lymphoma, whether this would be the result of their potential endocrine disrupting effect has not been investigated as yet. We explored the role of occupational exposure to endocrine disruptors in lymphoma aetiology.

Methods The Epilymph study is a multicenter case-control study carried out in six European countries from 1998 to 2004. We evaluated 2,457 controls and 2,013 lymphoma cases and its subtypes. Information on occupational history was collected through face-to-face interviews. We applied a job-exposure matrix (JEM) for endocrine disrupting chemicals to assess occupational exposures (Brouwers et al. 2009). We evaluated exposure to ten chemical groups: polycyclic aromatic hydrocarbons, polychlorinated organic compounds, pesticides, phthalates, solvents, bisphenol-A, alkylphenolic compounds, brominated flame retardants, metals and a miscellaneous group.

Results Prevalence of ever occupationally exposed among controls ranged from 1\% (bisphenol-A) to $48 \%$ (solvents). Risks for non-Hodgkin lymphoma (NHL) and chronic lymphocytic leukaemia (CLL) were increased with cumulative exposure to endocrine disruptors among men $(\mathrm{OR}=1.20 \mathrm{CI} 95 \%: 1.04-1.38$ and 1.28 CI95\%:1.01-1.61, respectively). However, none of the individual chemical groups evaluated was associated with NHL or follicular lymphoma risk. For other subtypes such as CLL, multiple myeloma, Hodgkin lymphoma and T-cell neoplasms risks were increased with several exposures, including metals 
(arsenic and copper), solvents (toluene and xylene), flame retardants, and ethylene glycol ethers.

Conclusions Some endocrine disruptors may play a role in the aetiology of certain lymphoma subtypes. Limitations in interpreting our findings include time- and country-related changes in exposure not reflected by the JEM, multiple comparisons and nondifferential misclassification leading to the attenuation of estimates for binary exposures.

Additional authors: Nikolaus Becker, Paolo Boffetta, Paul Brennan, Lenka Foretova, Marc Maynadie, Anthony Staines, Alexandra Nieters

\section{OCCUPATIONAL AND GENETIC RISK FACTORS FOR MYELOPROLIFERATIVE NEOPLASMS (MPN): A CASE- CONTROL STUDY}

CG Gross-Davis, Burstyn, Heavner, Klotz, Lynch, Newschaffer, Santella, Frank. Drexel Univeristy, Philadelphia, United States of America

\subsection{6/oemed-2013-101717.382}

Objectives The aetiology of a rare category of myeloproliferative neoplasms (MPN), bone marrow diseases with an excess of blood cells, is currently unknown. An MPN cluster in northeastern Pennsylvania allowed investigation of occupational risk factors and gene-environment interactions. Among our hypotheses were risks associated with aromatic and heterocyclic amines.

Methods This 2011 population-based case-control study assessed lifetime occupational, residential, smoking and dietary history by telephone interview. Cases $(n=55)$ were identified from the Pennsylvania cancer registry and a previous MPN study. Controls ( $\mathrm{n}=$ 473) were selected based on eligibility screening using random digit dialling. People born from 1921-1968 and residing in 3 counties with high incidence of MPN were eligible. Blood samples for genotyping were collected from 31 cases and 292 controls.

Results Cases were older (median age $=71$ vs $61 \mathrm{yrs}$ ) and more likely to be male (49\% vs 39\%) compared to controls but otherwise demographically similar. Ever working in ten employment areas (welding, painting, degreasing, firefighting or working with glue, solvents/inks, pesticides, diesel equipment, animals, or Xrays/radioactive material at the 8 most recent jobs) were not associated with MPN.

In analyses that examined the main effects of over 50 environmentally sensitive genes, the presence of NAT2 slow acetylator genotype, GSTM1 gene deletion, and GSTA1, and GSTM3 variants were associated with an increased risk for MPNs (unadjusted ORs 2.1-3.2, 95\% C. I.s excluding 1.0). Results were similar for analyses restricted to JAK2 positive cases.

Conclusions No relationship was found with occupations with presumed exposure to aromatic and heterocyclic amines, but our findings suggest that genotypes that modify the toxicity of these exposures may play a role in MPNs. Sources of exposures important to the pathway whereby NAT2 or other genotypes modify the effect of exposures in this population remain unclear and there is ongoing work on refining exposure assessment in the project.

\section{Session: 31. Exposure assessment IV}
383 DIFFERENT APPROACHES TO ESTIMATE EXPOSURE TO WORK STRESSORS, USING REPEATED MEASUREMENTS, AND THE ASSOCIATION WITH CARDIOVASCULAR DISEASE

\section{K Szerencsi, van Amelsvoort, Prins, Kant. Maastricht University, Maastricht, Nederland}

\subsection{6/oemed-2013-101717.383}

Objectives To explore the stability of exposure to work stressors over time and to examine the impact of different approaches of estimating exposure on the cardiovascular disease (CVD) risk estimation.

Methods The Maastricht Cohort Study-Work Stressor Score (MCS-WSS) was used to assess work stressors at three consecutive time points among 6154 employees participating in the MCS. Incident CVD was assessed with questionnaires. Five approaches were used to estimate exposure as: e.g. single exposure assessment, cumulative exposure above a cutoff point, total exposure and average exposure. The association between work stressors and CVD was stepwise adjusted for age, gender, educational level, smoking, body mass index, hypertension, leisure physical activity and negative affectivity.

Results The correlation between the work stressor scores assessed at the first and third time point was 0.58. Employees with a stable exposure above the highest quartile had a fully adjusted HR of 1.58 (95\% CI: 0.93-2.72). Employees with the highest quartile total exposure had a fully adjusted HR of 1.22 (95\% CI: 0.77-1.95) as compared to employees with the lowest quartile dose. Employees in the upper quartile of the average MCS-WSS had a fully adjusted HR of 1.26 (95\% CI: 0.79-2.01) as compared to employees in the lowest quartile of the MCSWSS. Employees with a single exposure assessment had a fully adjusted HR of 0.91 (95\% CI: 0.62-1.33).

Conclusions Employees with a stable exposure above the highest quartile score during a minimum of two years have the highest relative CVD risk.

\section{\begin{tabular}{|l|l}
384 & SKIN PHYSIOLOGY-BASED EVENTS IN
\end{tabular} THERMOREGULATION FOLLOWING EXPOSURE TO SIMULATED SOLAR RADIANT HEAT}

C P Chen, Chang, Chen. China Medical University, Taichung, Taiwan

\subsection{6/oemed-2013-101717.384}

Objectives The exposure to solar radiant heat (SRH) presents a risk of thermal stress. The risk is exacerbated when people have to experience an instantaneous change in the thermal environment in form of a temperature step due to loss of SRH, e.g., when walking into a thermal transient near a building entrance. This study investigated the skin physiology-based thermoregulation in response to SRH exposure and that occurred when experiencing a temperature step.

Methods The study was conducted in twin climate-controlled chambers, with the first chamber simulating a thermal exposure involving SRH (the outdoor chamber) and the second an environment maintained at a constant $24^{\circ} \mathrm{C}$ without SRH exposure (the indoor chamber). The temperature in the outdoor chamber was $28^{\circ} \mathrm{C}$, with the $\mathrm{SRH}$ present being equivalent to an increase in globe temperature of $4^{\circ} \mathrm{C}$. Ten male and female participants each first sat in the outdoor chamber for $30 \mathrm{~min}$ and were monitored for change in skin capillary blood flow (SCBF), skin moisture, and transepidermal water loss (TEWL), and then moved swiftly into the indoor chamber and were monitored for another $30 \mathrm{~min}$.

Results Following SRH exposure the SCBF increased in females but not in males. When experiencing the temperature drop, the $\mathrm{SCBF}$ required a longer period to decrease than the reduction 